\title{
NUMERICAL INVESTIGATION OF COOLING IN THE CONTINUOUS FIBER GLASS DRAWING PROCESS
}

\author{
Quentin Chouffart, ${ }^{1}$ Philippe Simon, ${ }^{2}$ Vincent E. Terrapon ${ }^{1, *}$ \\ ${ }^{1}$ Department of Aerospace and Mechanical Engineering, University of Liege, 4000 Liège, Belgium \\ 23B - The Fibreglass Company, 4651 Battice, Belgium
}

\begin{abstract}
The manufacturing process of glass fibers used for the reinforcement of composite material consists in drawing a glass melt at high temperature through an array of thousands of small orifices (i.e., the bushing plate) into fibers using a winder. This process is sensitive to numerous disturbances that can cause a fiber to break during the drawing process. This paper analyzes how the stress in the fiber depends on the controlling parameters of the process. The approach relies on numerical simulations and sensitivity analysis. Both a semi-analytical one-dimensional model and a more complex two-dimensional axisymmetric model are used. It is first found that radial variations across the fiber are small compared to changes in the axial direction and that the onedimensional approximation is accurate enough to describe the major trends in the process. Sensitivity analyses on some physical parameters controlling the heat transfers and on process parameters are then performed to identify strategies to reduce the axial stress. In particular, it is shown that, for a given fiber diameter, the stress is minimized if the glass melt temperature and the drawing velocity are increased. This approach is then applied to quantify the effect of inhomogeneous heat patterns on a bushing plate with a large number of fibers.
\end{abstract}

KEY WORDS: Manufacturing, Materials and food processing, Radiation, Convection, Glass fiber drawing, Glass process simulation

\section{INTRODUCTION}

The industrial process to manufacture glass fibers consists in continuously drawing and cooling a glass melt into fibers. The liquid glass is delivered by an upstream furnace to the bushing positions, which consist of a metal container with thousands of small orifices in the plate situated on its lower side. These holes have a tube shape with a diameter of 1 to $2 \mathrm{~mm}$, and are hereafter referred to as "tip" (see Figure 1). The typical number of tips varies from 800 to 8000 . Once the melt has flowed through these orifices, the liquid glass is transformed into a free jet which is quickly cooled to reach the glass transition and then wound at high velocity. The diameter of the final fiber is between 6.5 and $34 \mu \mathrm{m}$ depending on the product. During this process, a forming fiber may break, which leads to the failure of all fibers of the same bushing position. As a result, the position must be taken off-line for several minutes and a large quantity of glass is wasted, which can amount to up to $20 \%$ of the total glass production. The origins of the fiber breaking are multiple, such as a stone from refractories, gaz bubbles or other heterogeneity convected in the glass melt, thermal effects on the bushing plate leading to a too low or too high viscosity, instabilities... However, their underlying physical mechanisms are not totally understood. Since the efficiency of the manufacturing process is strongly limited by the fiber breakage, the overall objective of the present research is to characterize the physical effects that lead to such a failure, and

${ }^{*}$ Corresponding Vincent E. Terrapon: vincent.terrapon@ulg.ac.be 
to identify a strategy to reduce it. As a first step, the physics of the drawing process for a single filament is investigated both numerically and experimentally.

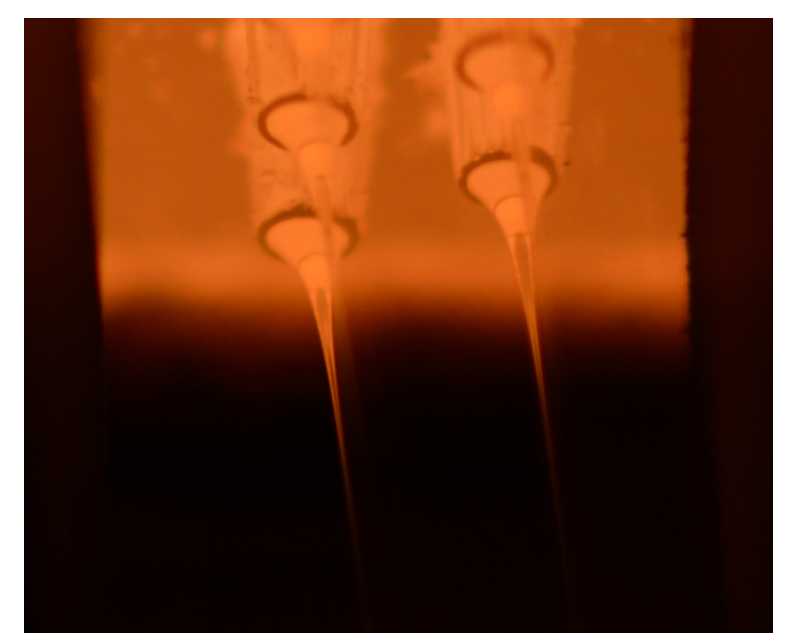

Fig. 1 View of the bushing plate including four tips and the corresponding forming fibers.

Different studies have already been performed on the physics of fiber glass drawing, but unfortunately many of the results still remain confidential within industrial companies. During the 1960's, Glicksman [1] used a onedimensional Newtonian fluid model to understand the behavior of the fiber during the manufacturing process. Neglecting two-dimensional effects was justified by the very small slope of the free surface in the central region, i.e. far away from the tip. His theorical study was compared to experimental data and good agreement was found, thus validating the one-dimensional assumption for the central region. However, this simplification is not adequate to accurately represent the region very close to the tip. Huynh and Tanner [3] solved numerically the two dimensional problem using a finite element method. They showed that radiation is the dominant mode of cooling in the vicinity of the tip exit and they proposed to use a non-constant effective emissivity in order to take into account the radius attenuation along the axial direction. Purnode $[4,5]$ also used a two-dimensional finite element model and relied on both steady and unsteady simulations. His results confirmed that radiation is the main heat transfer mechanism near the tip exit, while convection becomes dominant further away. Von der Ohe [6] performed a numerical parametric study for different glass types and process conditions as drawing speed, melt temperature and surrounding air. She pointed out that the drawing speed and cooling conditions of the surrounding air are important, in particular for the final product properties. Unfortunately, the results obtained cannot be directly applied in industrial production.

On the other hand, the experimental study by Rekhson [7] on a bushing plate with about hundred holes indicates that fiber break is mostly due to inhomogeneous heat patterns on the bushing plate. More recently, Liu [9] investigated numerically the variations of the final fiber diameter due to variabilities in the process and identified the temperature of the ambient air and of the furnace as their main cause. These findings led to the development of a control system to reduce the variations of the fiber diameter by adjusting the winder velocity. Finally, Chouffart et al. [13] have characterized numerically the most important factors impacting the cooling rate of the fiber. They have also studied the fiber stress variations in several cases of realistic process conditions.

Since Purnode [4], studies have been largely focused on the development of a physical two-dimensional axisymmetric model and on its numerical resolution. The good agreement between numerical solutions and experimental data found by many authors seems to indicate that such a model is sufficient to accurately describe the process. However, it has not yet been used to improve the robustness of the process. The industrial production can draw thousands of fibers on the same bushing position. As a result, each fiber is formed under varying and often sub-optimal conditions. This is particularly true regarding the tip temperature. To date, no numerical study has taken into account the typical variability occurring in the process, and which is observed 
to be critical.

The goal of this paper is to fully exploit such a model to understand the physics controlling the forming of the fiber and to identify the key process parameters. The influence of different physical parameters controlling heat transfers and several realistic industrial conditions are analyzed. Since the ultimate objective is to reduce the fiber break, the different sensitivity analyses focus on the axial stress.

Additionally, as many simulations are required, a semi-analytical one-dimensional model based on Glicksman's model [1] is also considered. Although its solution is essentially analytical, a numerical treatment is still required to obtain the full solution. Nevertheless, it has the advantage of being much faster than the full two-dimensional model with a small impact on the solution accuracy, at least in terms of the quantities of interest here. Additionally, the semi-analytical model explicitly demonstrates the influence of some physical parameters.

This paper is organized as follows. The physical two-dimensional model and its mathematical formulation is described in section 2, while the one-dimensional approximation is summarized in section 3. Section 4 presents the results. First, the accuracy of the one-dimensional model is assessed in 4.1. The sensitivity of heat transfers on physical parameters is then presented in 4.2. Finally, sections 4.3 and 4.4 analyze the influence of the process conditions and of the spatial variations of the bushing plate temperature when many fibers are considered. Conclusions and future work can be found in section 5 .

\section{TWO-DIMENSIONAL AXISYMMETRIC MODEL}

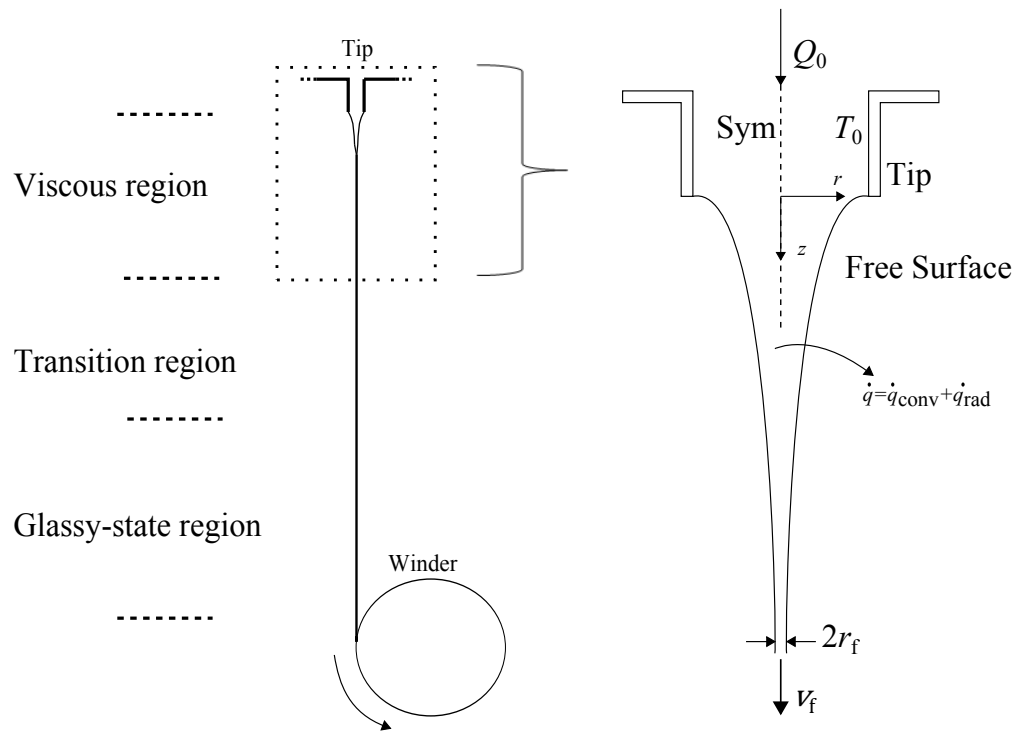

Fig. 2 Schematics of the drawing process for a single fiber with the computational domain (indicated by the dotted frame on the left) and boundary conditions.

Glass fiber drawing involves various complex physics including the transition from liquid to solid state. Based on this, the fiber can be divided into three main regions, as illustrated in Figure 2. The first region is situated near the tip exit where the glass is still in the liquid phase and can be considered as a Newtonian viscous fluid. The heat transfers with the surrounding environment are dominated by radiation and convection. This region has the particularity of exhibiting a cone shape, which results from the strong acceleration of the glass melt. The second region is located around the transition point where the glass behaves like a viscoelastic material. The radiation becomes negligible because of the much lower fiber temperature and the cooling is mostly due to convection. Except for temperature, little change in the physical properties of the fiber is observed. Finally, the last region corresponds to the part of the fiber where the glass is in a solid state. Note that the defining 
characteristics of these three regions change smoothly and continuously, so that their boundaries are only qualitative. This work focuses on the first region, i.e. where the glass is in the liquid state, because most of the variations of the key quantities occur in this region.

\section{Governing equations}

The model used is based on following assumptions: the glass material is modeled as an incompressible Newtonian viscous fluid, the flow is assumed to be axisymmetric, and only the steady state is considered. The liquid glass can be described by the basic governing equations of mass, momentum and energy conservation:

$$
\begin{aligned}
& \boldsymbol{\nabla} \cdot \mathbf{v}=0, \\
& \rho(\mathbf{v} \cdot \boldsymbol{\nabla}) \mathbf{v}=\boldsymbol{\nabla} \cdot \boldsymbol{\tau}, \\
& \rho c_{p} \mathbf{v} \cdot \boldsymbol{\nabla} T=\boldsymbol{\tau}: \nabla \mathbf{v}+\boldsymbol{\nabla} \cdot(k \boldsymbol{\nabla} T)-\boldsymbol{\nabla} \cdot \dot{\mathbf{q}}_{\mathrm{r}},
\end{aligned}
$$

where $\mathbf{v}$ is the velocity vector, $T$ the temperature, $\boldsymbol{\tau}$ the stress tensor, and $\dot{\mathbf{q}}_{\mathrm{r}}$ the radiative heat flux. The material considered here is the Advantex $®$ glass ${ }^{1}$. The density $\rho$, the specific heat capacity $c_{p}$ and the conductivity $k$ are considered constant as their temperature dependence for this glass is weak in the temperature range considered (this assumption is further discussed in section 4). For a Newtonian fluid the stress tensor is equal to $\boldsymbol{\tau}=-p \mathbf{I}+2 \eta \mathbf{D}$, where $p$ is the isotropic pressure and $\mathbf{D}=1 / 2\left(\boldsymbol{\nabla} \mathbf{v}+\boldsymbol{\nabla} \mathbf{v}^{\mathrm{T}}\right)$ the strain-rate tensor. The dependence of the viscosity $\eta$ on the temperature $T$ is given by Fulcher's law:

$$
\eta=10^{-A+\frac{B}{T-T_{0}}}
$$

where $A, B$ and $T_{0}$ are three constants that depend on the material. The temperature interval considered in the present simulations ranges from $1300^{\circ} \mathrm{C}$ to $800^{\circ} \mathrm{C}$, which leads to viscosity variations of more than ten orders of magnitude. Note that the velocity and stress fields only depend on the temperature through the viscosity. The glass melt is a semi-transparent medium for some range of wavelengths. Therefore, not only conduction but also internal radiation contribute to internal heat transfers. An accurate radiation model would be required to obtain the precise internal temperature profile and surface radiative flux. However, this effect is neglected here and the radiative internal heat flux $\dot{\mathbf{q}}_{\mathrm{r}}$ in Eq. (3) is set to zero.

\section{Boundary conditions}

A two-dimensional axisymmetric domain is considered, which includes the fiber and the glass flow inside the tip. At the tip inlet, the volumetric flow rate $Q_{0}$ is imposed based on the height $H$ of the glass column above it and the viscosity of the melt [10]:

$$
Q_{0}=\frac{\pi}{8 \eta}\left(\frac{\mathrm{d} p}{\mathrm{~d} z}\right) r_{0}^{4}=\frac{\pi}{8 \eta}\left(\frac{\rho g H}{L}\right) r_{0}^{4}
$$

where $\mathrm{d} p / \mathrm{d} z$ is the static pressure gradient, $g$ the gravity constant and $r_{0}$ and $L$ are the tip radius and length, respectively. In practice, the viscosity at the tip should be approximately 1000 Poise to ensure fiberization, which corresponds to a temperature called $T_{3}$ (since $\log _{10} \eta=3$ ). Therefore, a temperature $T_{0}$ around the value of $T_{3}$ (generally between $\log _{10} \eta=2.5$ and $\log _{10} \eta=3$ ) is used as boundary condition for the tip walls, where a no-slip condition is imposed for the velocity field. At the outlet, a constant drawing velocity $v_{\mathrm{f}}$ is imposed in the axial direction. The final fiber diameter is thus directly controlled by the inlet volumetric flow rate and the drawing velocity.

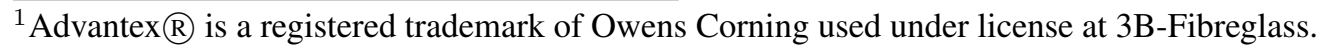


Along the free surface, the interface condition is given by

$$
\begin{gathered}
\mathbf{n} \cdot \boldsymbol{\tau}_{\mathrm{g}}=\gamma \mathbf{n}(\boldsymbol{\nabla} \cdot \mathbf{n}), \\
\mathbf{v} \cdot \mathbf{n}=0,
\end{gathered}
$$

where $\gamma$ is the surface tension assumed constant, $\boldsymbol{\tau}_{\mathrm{g}}$ the interface stress for glass, and $\mathbf{n}$ the surface normal. Note that the stress interface for the air is neglected here.

Both convective and radiative heat losses take place at the free surface. The heat flux leaving the fiber can thus be written as

$$
\dot{q}_{\mathrm{s}}=\dot{q}_{\mathrm{conv}}+\dot{q}_{\mathrm{rad}}=h\left(T_{\mathrm{s}}-T_{\text {ext,conv }}\right)+\epsilon \sigma\left(T_{\mathrm{s}}^{4}-T_{\text {ext,rad }}^{4}\right),
$$

where $h$ is the convective heat transfer coefficient, $T_{\mathrm{s}}$ the surface temperature, $T_{\text {ext,conv }}$ the ambient air temperature around the fiber for convection, $T_{\text {ext,rad }}$ the surrounding temperature for radiation, $\epsilon$ the emissivity of the fiber surface and $\sigma$ the Stefan-Boltzmann constant. The convective cooling is induced by the high drawing velocity of the fiber (of the order of $20 \mathrm{~m} / \mathrm{s}$ ). The air is heated in the vicinity of the bushing plate (whose temperature is around $T_{3}$ ) and is entrained downwards by the fiber. As a result, experimental measurements show a non-constant value for $T_{\text {ext,conv }}$ along the fiber as discussed below. $T_{\text {ext,rad }}$ is considered constant and generally set to $25^{\circ} \mathrm{C}$ for the case of a single fiber.

The convective heat transfer coefficient $h$ is approximated by Kase and Matsuo correlation for a thin cylinder moving axially [11]:

$$
h=0.42 \frac{k_{\mathrm{a}}}{2 r} \operatorname{Re}^{0.334},
$$

where $k_{\mathrm{a}}$ is the air conductivity and $r(z)$ the fiber radius, and the Reynolds number is defined as $\operatorname{Re}=v_{z} 2 r / v_{\mathrm{a}}$, with $v_{\mathrm{a}}$ and $v_{z}$ the air kinematic viscosity and the fiber axial velocity at the surface, respectively. This relation has been used in many previous studies and seems to be adequate $[4,6,7]$.

The governing equations are solved numerically. The numerical solver is ANSYS POLYFLOW [14] using a finite-element computational fluid dynamic method. The free surface is treated with an Arbitrary LagrangianEulerian (ALE) formulation. In order to ensure a grid-independent solution, the number of nodes in each direction has been doubled. The resulting solution shows a maximum variation of $0.1 \%$ compared to the initial case. Additionally, the global conservation of mass, momentum and energy has been verified over the entire computational domain.

\section{ONE-DIMENSIONAL SEMI-ANALYTICAL MODEL}

The model described above can only be solved numerically. Nevertheless, an analytical solution can be found if several simplifications are made. The resulting formulation is useful to understand the dependencies between all the parameters in the model. The other advantage of this approach is the reduced computational cost compared to the 2D model. Hence, a higher number of case studies can be performed. The model is based on Glicksman's [1] and considers a uniaxial extensional Newtonian flow where all variables depend only on the axial direction $z$. For simplification, the surface tension is neglected.

Conservation of mass in the axial direction leads to

$$
\rho v(z) \pi r(z)^{2}=\rho Q_{0}=\text { const },
$$


while conservation of momentum at steady state gives

$$
F(z)=\tau_{z z}(z) \pi r(z)^{2}=\rho Q_{0} v_{z}-\text { const },
$$

where $\tau_{z z}$ is the total stress (i.e., including the isotropic normal component) and $F(z)$ the axial force.

For a uniaxial extensional Newtonian flow, the total stress can be approximated in the region away from the cone by

$$
\tau_{z z}=-p+2 \eta \frac{\partial v_{z}}{\partial z}=\eta_{\mathrm{e}} \frac{\mathrm{d} v_{z}}{\mathrm{~d} z}
$$

where $\eta_{\mathrm{e}}(z)=3 \eta(z)$ is the extensional viscosity. Note that the viscosity also depends on $z$ through equation (4) such that $\eta(z)=\eta(T(z))$. For the specific conditions considered here, the axial force $F(z)$ is approximately constant along the fiber (see also [2]), which leads to

$$
F(z)=\pi r(z)^{2} 3 \eta(z) \frac{\mathrm{d} v_{z}}{\mathrm{~d} z}=3 Q_{0} \eta(z) \frac{\mathrm{d} \ln v_{z}}{\mathrm{~d} z} \approx \mathrm{const} \equiv F .
$$

This relation can be integrated to yield

$$
v_{z}(z)=v_{0} \exp [\frac{F}{3 Q_{0}} \underbrace{\int_{0}^{z} \frac{1}{\eta\left(z^{\prime}\right)} \mathrm{d} z^{\prime}}_{\equiv \varphi(z)}],
$$

where $v_{0}=Q_{0} /\left(\pi r_{0}^{2}\right)$ is the velocity at the tip. Evaluating expression (14) at the coordinate where the drawing force is applied, $z=z_{\mathrm{f}}$, one obtains

$$
F=\frac{3 Q_{0}}{\varphi_{\mathrm{g}}} \ln \left(\frac{v_{\mathrm{f}}}{v_{0}}\right)
$$

with

$$
\varphi_{\mathrm{g}}=\varphi\left(z_{\mathrm{f}}\right)=\int_{0}^{z_{\mathrm{f}}} \frac{1}{\eta\left(z^{\prime}\right)} \mathrm{d} z^{\prime}
$$

Expression (15) for $F$ can now be used to obtain

$$
\begin{aligned}
v_{z}(z) & =v_{0}\left(\frac{v_{\mathrm{f}}}{v_{0}}\right)^{\varphi(z) / \varphi_{\mathrm{g}}}, \\
r(z) & =r_{0}\left(\frac{v_{\mathrm{f}}}{v_{0}}\right)^{-\varphi(z) / 2 \varphi_{\mathrm{g}}}, \\
\tau_{z z}(z) & =\frac{3}{\varphi_{\mathrm{g}}} v_{z}(z) \ln \left(\frac{v_{\mathrm{f}}}{v_{0}}\right) .
\end{aligned}
$$

The integral $\varphi(z)$ represents the variation of viscosity along the fiber. Since the glass viscosity behaves according to Fulcher's law (4), its value is highly dependent on the temperature field, especially in the lower temperature range. Consequently, a small variation in the fiber temperature generates a large impact on the viscosity, and thereby on the flow. The integrals $\varphi(z)$ and $\varphi_{\mathrm{g}}$ are the only link between the flow dynamics and the heat transfers. They require to solve the energy equation (3) to obtain the temperature profile:

$$
\frac{\mathrm{d} T(z)}{\mathrm{d} z}=-\frac{2 \pi r_{0}}{\rho c_{p} Q_{0}} \dot{q}_{\mathrm{s}}(z)\left(\frac{v_{\mathrm{f}}}{v_{0}}\right)^{-\varphi(z) / 2 \varphi_{\mathrm{g}}},
$$

where $\dot{q}_{\mathrm{s}}(z)$ is given by (8) with $T_{\mathrm{s}}=T(z)$. 


\begin{tabular}{lccccc}
\hline \hline & $Q_{0}\left[\mathrm{~m}^{3} / \mathrm{s}\right]$ & $r_{0}[\mathrm{~m}]$ & $H[\mathrm{~m}]$ & $T_{0}\left[{ }^{\circ} \mathrm{C}\right]$ & $v_{\mathrm{f}}[\mathrm{m} / \mathrm{s}]$ \\
\hline Base case & $2.1255 \cdot 10^{-9}$ & $0.66 \cdot 10^{-3}$ & 0.3 & 1300 & 27.1 \\
Sensitivity analysis & - & {$[0.55-0.86] \cdot 10^{-3}$} & {$[0.15-0.87]$} & {$[1253-1382]$} & {$[9.4-55.3]$} \\
\hline \hline
\end{tabular}

Table 1 Process parameters used in this study and ranges of variation for the sensitivity analysis: volumetric flow rate $Q_{0}$, tip radius $r_{0}$, height $H$ of the glass column, tip temperature $T_{0}$, and drawing velocity $v_{\mathrm{f}}$.

The solution of the one-dimensional model is not entirely analytical since equation (20) requires a numerical solution. This is solved with a simple finite difference discretization for the axial temperature derivative. Because each variable depends on the integral over the entire computational domain, $\varphi_{\mathrm{g}}$, an iterative method is necessary. An arbitrary value for $\varphi_{\mathrm{g}}$ is first chosen, equation (20) is then solved numerically and a new guess for $\varphi_{\mathrm{g}}$ is computed. The procedure is repeated until convergence is reached.

\section{RESULTS AND DISCUSSION}

\subsection{General solution and comparison between the semi-analytical model and the 2D model}

The 2D model has already been validated with two different sets of experimental data in a previous study [13]. The solution for the radius profile along the axial component was compared with experimental data from both the literature and measurements provided by an in-house experimental unit. For both cases, the data of the radius have been extracted from picture of the forming zone. Good agreement was found in each case. Unfortunately, the radius is the only parameter which can be experimentally measured due to the very different and small scales of the forming fiber. The aim here is to compare the solution obtained with the simpler onedimensional model (section 3) with that of the axisymmetric model (section 2). The chosen test case is based on process conditions very similar to the industrial production of $10 \mu \mathrm{m}$ fibers, as summarized in Table 1 . $T_{\text {ext,conv }}$ is considered constant for this test case and is set to $600^{\circ} \mathrm{C}$ as a first approximation (see below).

Figure 3a shows the axial temperature profile along the fiber and illustrates the rapid cooling of the glass during the process, as the temperature decreases by approximately $500^{\circ} \mathrm{C}$ over a distance of $8 \mathrm{~cm}$. Moreover, the cooling is most intense in the region near the tip where heat losses are mainly due to radiation (i.e., the region of steepest slope in Figure 3a, from $z=0$ to $z=0.005 \mathrm{~m}$ ). This cooling is also compounded by the decrease of the fiber radius. As the temperature decreases, the viscosity and, thus, the velocity strongly increase. As a result of mass conservation, a strong attenuation of the fiber radius is observed, as illustrated by the well-known conical shape of the forming fiber (see Figure 1). Note that radius attenuation is mostly driven by non-isothermal effects and is thereby exacerbated by large cooling rates. After about $5 \mathrm{~mm}$, heat transfers are then mainly controlled by convection [13]. Figure 4a shows that the velocity and, thus, the radius converge to a constant value at a distance of about 3 to $4 \mathrm{~cm}$ from the tip.

In order to assess the importance of the radial variations across the fiber (i.e., two-dimensional effects), axial profiles along both the free surface and the centerline obtained from the 2D model are plotted in Figures 3a and 4a. These profiles are also compared with the profiles obtained from the one-dimensional model. The relative variations across the fiber and the relative discrepancies between the 2D and 1D models are very small, in particular for the velocity. This is more clearly seen in Figures $3 b$ and $4 b$, which show the actual difference between these different profiles. The temperature difference between the free surface and the symmetry line reaches a maximum of $16^{\circ} \mathrm{C}$ very close to the tip, while a maximal difference of $20^{\circ} \mathrm{C}$ is observed with the 1D model. This difference is very small compared to the absolute magnitude of the temperature and to the overall temperature range. Radial variations of the velocity are even smaller, as demonstrated by Figure 4b. This can be explained by the free surface condition imposed, which leads to a very flat radial velocity profile. The small velocity variations are solely due to variations of the viscosity, themselves driven by the small radial temperature variations. 


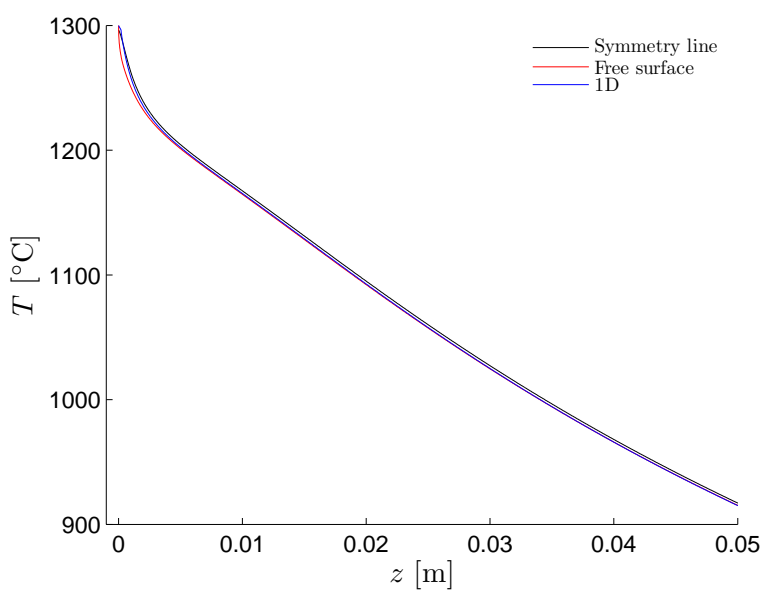

(a)

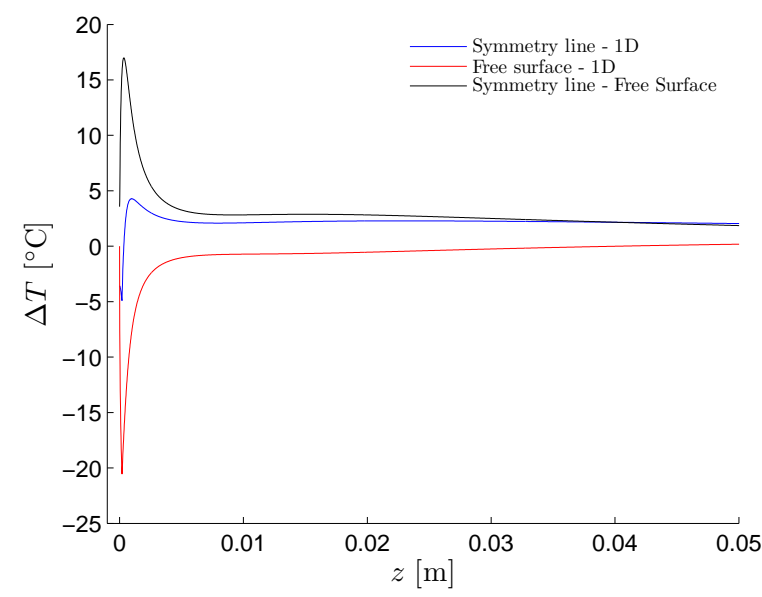

(b)

Fig. 3 (a) Axial temperature profile $T(z)$ as a function of the distance $z$ from the tip for the axisymmetric 2D and extensional 1D model (blue); for the 2D model, profiles are taken along both the free surface (red) and the symmetry line (black). (b) Difference of temperature $T(z)$ between the solution from the 2D model and the 1D model at the symmetry line (blue) and at the free surface (red); the difference between the two profiles from the $2 \mathrm{D}$ model is also represented (black).

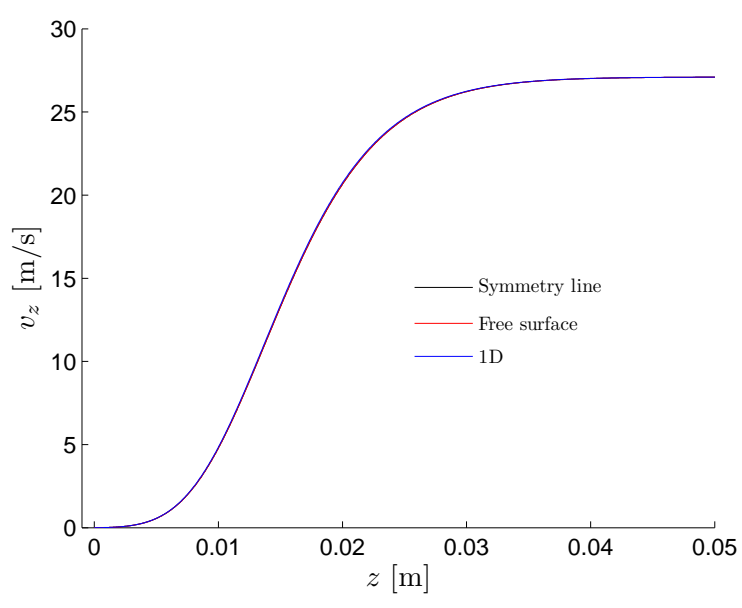

(a)

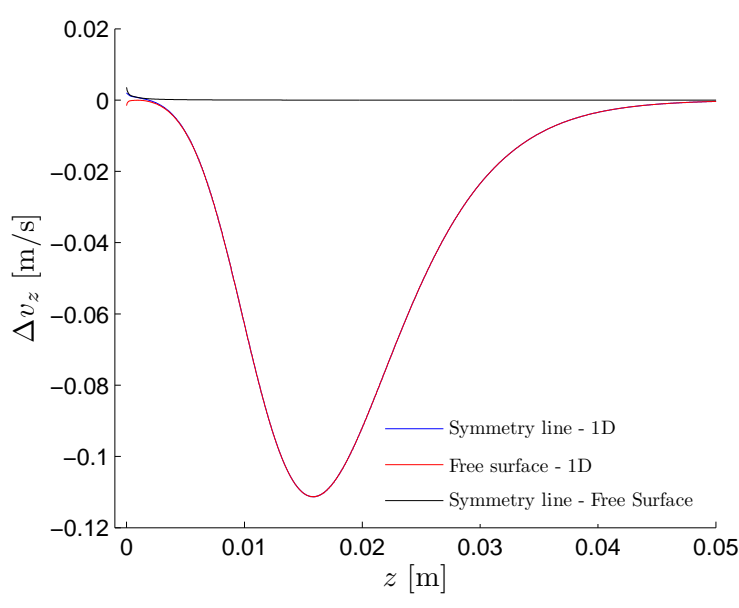

(b)

Fig. 4 (a) Axial velocity profile $v_{z}(z)$ as a function of the distance $z$ from the tip for the axisymmetric 2D and extensional 1D model (blue); for the 2D model, the profiles are taken along both the free surface (red) and the symmetry line (black). (b) Difference of velocity $v_{z}(z)$ between the solution from the 2D model and the 1D model at the symmetry line (blue) and at the free surface (red); the difference between the two profiles from the $2 \mathrm{D}$ model is also represented (black). 
In conclusion, neglecting radial variations (i.e, two-dimensional effects) leads to relative errors on the temperature of the order of $1.2 \%$ with a maximum of $4 \%$ very close to the tip. The final axial stress, which is used as main quantity of interest in this study, shows a deviation of about $1 \%$ at the fiber surface to about $10 \%$ at the centerline (not shown). Although the discrepancy is not negligible at the centerline, the maximum stress, which is the relevant quantity, is situated at the surface where the one-dimensional approximation is quite accurate. Finally, it can be demonstrated using the 2D model that the force $F$ in this region varies only slightly ( $\sim 3 \%$ ), which is consistent with Von Der Ohe [6].

In light of the many other approximations made, these error levels are deemed acceptable to study the relative influence of the different parameters and to optimize the operational window. Moreover, the semi-analytical approach is computationally much more efficient and robust. Nonetheless, one has to keep its limitations in mind, especially in the region near the tip. Finally, the surface tension, neglected here, should be considered for unsteady cases as it can destabilize the drawing process [5].

2D simulations have also been used to assess the validity of the constant properties assumption. The relative variations of the density, specific heat and thermal conductivity over the entire temperature range are approximately $2 \%, 4.2 \%$ and $13.6 \%$, respectively. These variations lead to a relative difference of $1.7 \%, 3.7 \%$ and $1.5 \%$ in the final axial stress. In light of these small variations, the assumption of constant properties seems adequate.

\subsection{Sensitivity analysis of physical parameters controlling heat transfers}

The cooling of the fiber is due to both radiative and convective heat transfer mechanisms. Specifically, the heat flux leaving the fiber surface is given by (8). This flux is controlled by four different parameters: the convective coefficient $h$, the surrounding temperatures $T_{\text {ext,conv }}$ and $T_{\text {ext,rad }}$, and the emissivity $\epsilon$ of the fiber surface. The convective coefficient is the most complex parameter since it is related to the fiber radius, the fiber velocity, and the air properties. Considering equation (9) with (17) and (18), one has

$$
h=h_{0}\left(v_{0}\right)^{0.334}\left(r_{0}\right)^{-0.666}\left(\frac{v_{\mathrm{f}}}{v_{0}}\right)^{0.666 \frac{\varphi(z)}{\varphi_{\mathrm{g}}}}
$$

where $h_{0}=0.42 k_{\mathrm{a}} / \nu_{\mathrm{a}}^{0.334}$ depends on the air kinematic viscosity $v_{\mathrm{a}}$ and the air conductivity $k_{\mathrm{a}}$. As mentioned above, the radiative heat flux is larger than the convective heat flux in the region very close to the tip. Further away, as the temperature decreases, the convection rapidly becomes the main cooling mechanism.

In order to understand the impact of these mechanisms, the sensitivity of the heat flux and normal stress on the convective coefficient $h_{0}$ and radiation emissivity $\epsilon$ has been analyzed. The parameter $h_{0}$ characterizes the contribution of the surrounding environment properties. The range of variability of these two parameters is set to approximately $\pm 25 \%$. The air temperature is initially taken as a constant to remove any effect its variation may have, and the stress is chosen as quantity of interest since it controls directly the fiber breaking [8]. Figure 5 shows the final value of the axial stress (i.e., at the location where the drawing force is applied), which is defined as

$$
\tau_{z z, \mathrm{f}}=\frac{3}{\varphi_{\mathrm{g}}} v_{\mathrm{f}} \ln \left(\frac{v_{\mathrm{f}}}{v_{0}}\right) .
$$

For each parameter value, an increase in fiber cooling (i.e., larger $\epsilon$ or larger $h_{0}$ ) increases the final value of the stress. In this case, results show a greater variation of the stress when $\epsilon$ varies compared to $h_{0}$. As $z$ increases, the viscosity increases dramatically, and thus, from (16), its contribution to the integral $\varphi(z)$ becomes negligible. As a result, $\varphi(z)$ reaches a plateau after a few centimeters. From this observation and relation (19), it can be seen that the stress also exhibits a constant plateau given by (22) as both the velocity and $\varphi(z)$ become constant. A higher cooling leads to a larger viscosity variation, and thus to a lower $\varphi_{\mathrm{g}}$ and, from (22), to a larger stress. This also demonstrates that the final stress depends on the distribution of the heat 


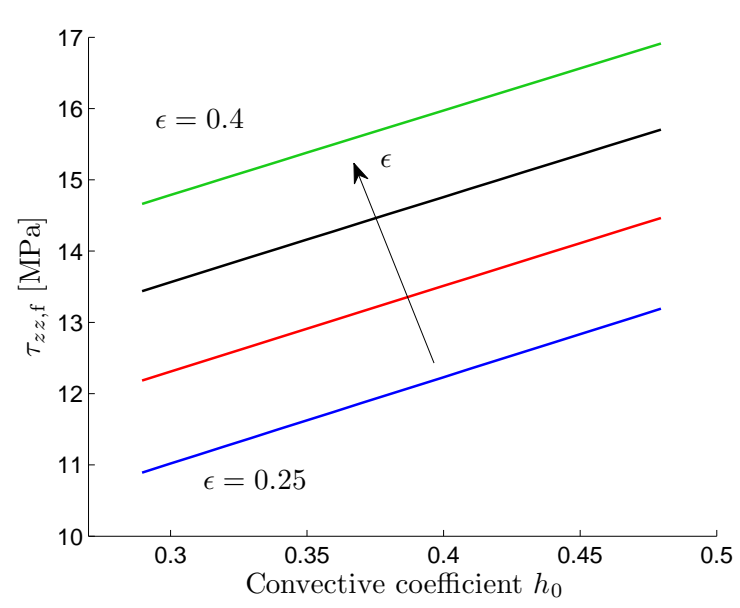

(a)

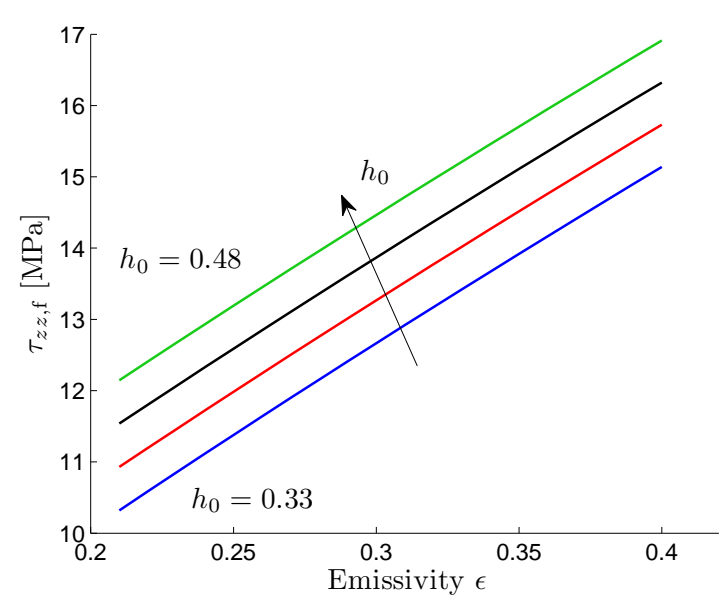

(b)

Fig. 5 (a) Final axial stress $\tau_{z z, \mathrm{f}}$ as a function of the convection coefficient $h_{0}$ from equation (21) for different values of the emissivity $\epsilon$. (b) Final axial stress $\tau_{z z, \mathrm{f}}$ as a function of the emissivity $\epsilon$ for different values of $h_{0}$. The extreme values of the respective parameter are indicated next to the blue and green lines.

flux and not on the final temperature since this temperature continues to decrease after the stress plateau has been reached. Additionally, the sensitivity of $\varphi_{\mathrm{g}}$ is larger at high temperature (i.e. at lower viscosity), which corresponds to the region close to the tip where radiation dominates. It is therefore conjectured that the stress is more affected by radiation than convection.

Many previous studies have considered a constant ambient air temperature. However, experimental measurements show a strong gradient of $T_{\text {ext,conv }}$ along the vertical coordinate $z$, due to the heating of the entrained air by the bushing plate. Figure 6a shows experimental measurements of the air temperature $T_{\text {ext,conv }}$ around a single fiber obtained on an in-house experimental test bench. The measured temperature can be interpolated by an exponential as

$$
T_{\text {ext,conv }}(z)=T_{\infty}+\left(T_{\mathrm{b}}-T_{\infty}\right) e^{-a z},
$$

where $T_{\infty}$ is the room ambient air temperature far away from the busing plate and $T_{\mathrm{b}}$ the air temperature at the tip. Note that temperature $T_{\text {ext,conv }}$ has been measured close to the fiber, but outside of its thermal boundary layer (which has a thickness of approximately $1 \mathrm{~mm}$ at $z=0.01 \mathrm{~m} \mathrm{[15]).} \mathrm{In} \mathrm{the} \mathrm{measurement} \mathrm{region,} T_{\text {ext,conv }}$ is constant over a radial distance of about $8 \mathrm{~cm}$ before decreasing to $T_{\infty}$ far away from the fiber, which shows that variations in $T_{\text {ext,conv }}$ stem from the heating at the bushing plate and not from the convection process itself. On the other hand, $T_{\text {ext,rad }}$ is assumed constant. Finally, it is important to mention that the correlation (9) has been developed for a constant external temperature, so that its use with a varying $T_{\text {ext,conv }}$ is expected to induce some error. However, this error is likely smaller than if variations of $T_{\text {ext,conv }}$ are neglected.

Similar results as above are obtained if the variations of the surrounding air temperature are considered: the larger the cooling rate, the larger the final stress. Variations in the final axial stress of the same order of magnitude as in the previous case are also observed when the parameters controlling the surrounding air temperature are varied, i.e., $\left(T_{\mathrm{b}}-T_{\infty}\right)$ and $a$ in equation (23), as illustrated in Figure 6b. Overall, the axial stress seems to increase with higher heat transfers at the fiber surface. A more gradual cooling could represent a strategy to reduce fiber breaking.

\subsection{Influence of the process parameters}

The efficiency of the process (e.g., low breaking rate) strongly depends on the operating window. This window is controlled by following process parameters: flow rate $Q_{0}$, tip temperature $T_{0}$ and drawing velocity $v_{\mathrm{f}}$. In turn, 


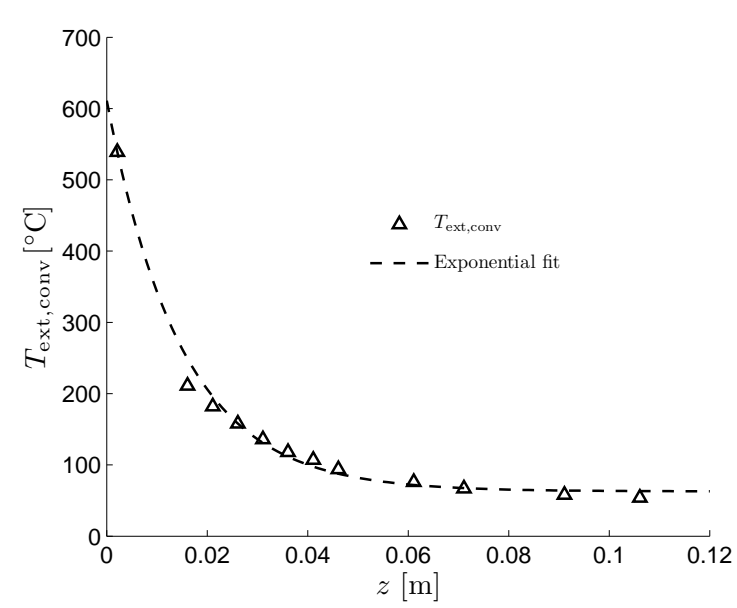

(a)

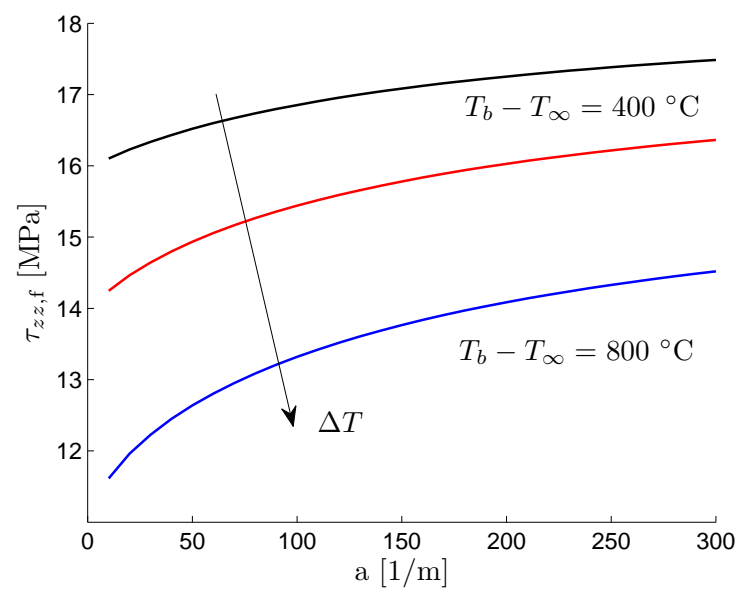

(b)

Fig. 6 (a) Measured air temperature $T_{\text {ext,conv }}$ in the surrounding environment of a single fiber as a function of the axial distance $z$ from the tip (symbols) and exponential fit (dashed line). (b) Final axial stress $\tau_{z z, \mathrm{f}}$ as a function of the coefficient $a$ from equation (23) for different values of $\Delta T=T_{\mathrm{b}}-T_{\infty}$.

the volumetric flow rate $Q_{0}$ depends on the glass height $H$ above the tip, the temperature $T_{0}$ (i.e., viscosity) of the glass at the tip and the tip geometry $r_{0}$ and $L$. In order to better understand how the definition of the operating window impacts the process, the one-dimensional model is used to study the sensitivity of the stress on four control parameters: $T_{0}, H, v_{\mathrm{f}}$ and $r_{0}$. The same test case is used as previously, except for $T_{\text {ext,rad }}$, which is set to $600^{\circ} \mathrm{C}$ to take into account the presence of finshields around the fiber.

In a first step, each parameter is varied within a given interval (as indicated in Table 1), while keeping the other parameters constant. These intervals have been defined such that the final fiber diameter ranges between 7 and $17 \mu \mathrm{m}$ for a nominal value of $10 \mu \mathrm{m}$. Figure $7 \mathrm{a}$ shows the final stress $\tau_{z z, \mathrm{f}}$ as a function of the inverse of $\varphi_{\mathrm{g}}$ (i.e. the average viscosity variation by unit of length) for the four parametric cases considered. One can observe that the parametric curves for $r_{0}, H$ and $T_{0}$ (i.e., controlling the flow rate) almost collapse onto one curve, which appears to be linear in $\varphi_{\mathrm{g}}^{-1}$. This can be explained by looking at equation (22), which shows that the final stress is, up to the factor $\ln \left(v_{\mathrm{f}} / v_{0}\right)$, linear in $\varphi_{\mathrm{g}}^{-1}$ (when $v_{\mathrm{f}}$ is kept constant). Despite changes in $v_{0}$ with the flow rate, the variations of the factor $\ln \left(v_{\mathrm{f}} / v_{0}\right)$ are very small, leading to this almost linear behavior. The largest range of axial stress, from $2 \mathrm{MPa}$ to $62 \mathrm{MPa}$, is obtained by varying $T_{0}$. This is due to the large sensitivity of $\varphi_{\mathrm{g}}$ on the viscosity near the tip, as already mentioned in the previous section. Temperature $T_{0}$ is thus a critical parameter in controlling the process. On the other hand, variations of $v_{\mathrm{f}}$ lead to small changes in $\varphi_{\mathrm{g}}$, as indicated by the almost vertical curve. In this case, the final stress is almost linear in $v_{\mathrm{f}}$. Figure $7 \mathrm{~b}$ represents the same data, but as a function of the final radius $r_{\mathrm{f}}$.

This previous analysis assumes that each of the four parameters can be changed independently to steer production. However, $r_{0}$ and $H$ are design parameters that cannot be modified during production. Moreover, the final radius is typically imposed by the customer. As a consequence, only $T_{0}$ are $v_{\mathrm{f}}$ are actual production control parameters. Furthermore, these two parameters are not independent since any variation of one without adjustment of the other would change the final fiber radius. This is illustrated in Figure 8 where $T_{0}$ is varied from $1250^{\circ} \mathrm{C}$ to $1310^{\circ} \mathrm{C}$ and $v_{\mathrm{f}}$ is adjusted to keep a constant final radius. Three typical diameters found in the industry are considered, i.e., 10, 12 and $17 \mu \mathrm{m}$. The chosen range of temperature leads to a decrease by a factor of two of the final stress across the entire interval.

It is clear that the stress level increases when the fiber diameter is reduced, explaining the difficulties encountered when drawing very thin fibers. Furthermore, this analysis also shows that the axial stress is minimized for 


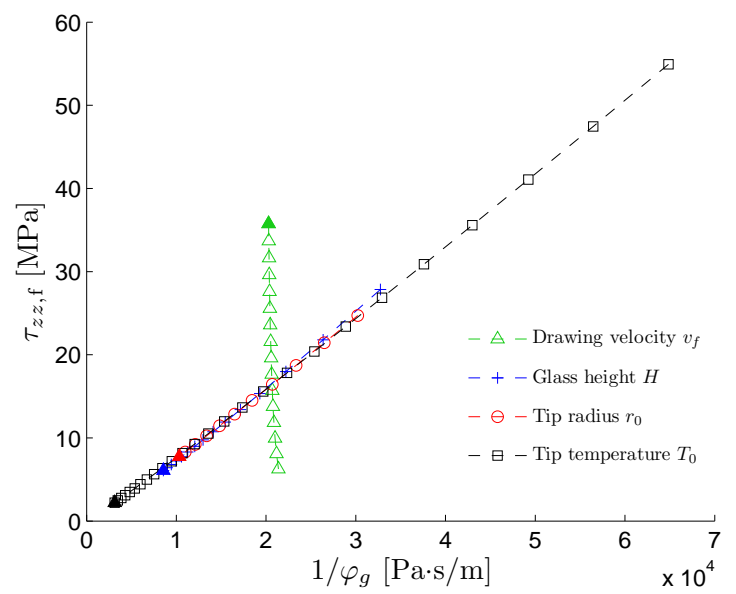

(a)

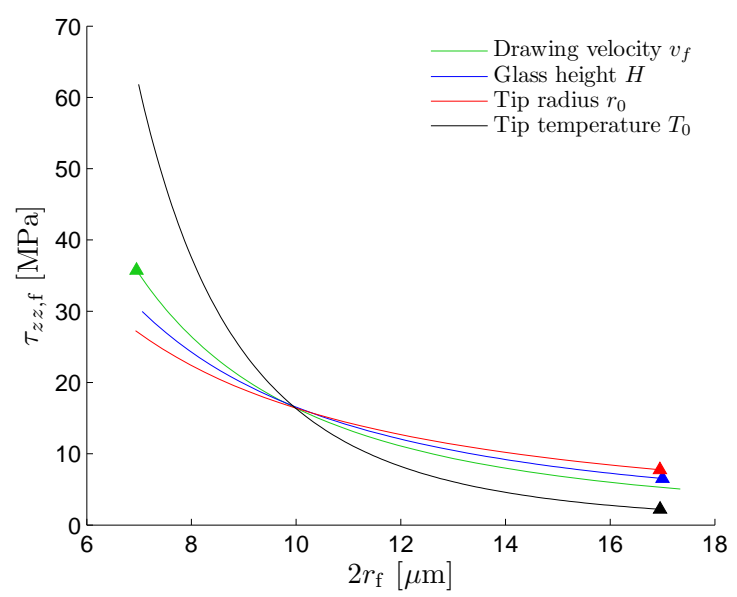

(b)

Fig. 7 Final axial stress $\tau_{z z \text {,f }}$ as a function of (a) $1 / \varphi_{g}$, and (b) the final radius $r_{\mathrm{f}}$, for four parametric cases. Each color corresponds to the variations of one parameter while keeping the others constant. The closed symbols correspond to the maximum value of the respective parameter.

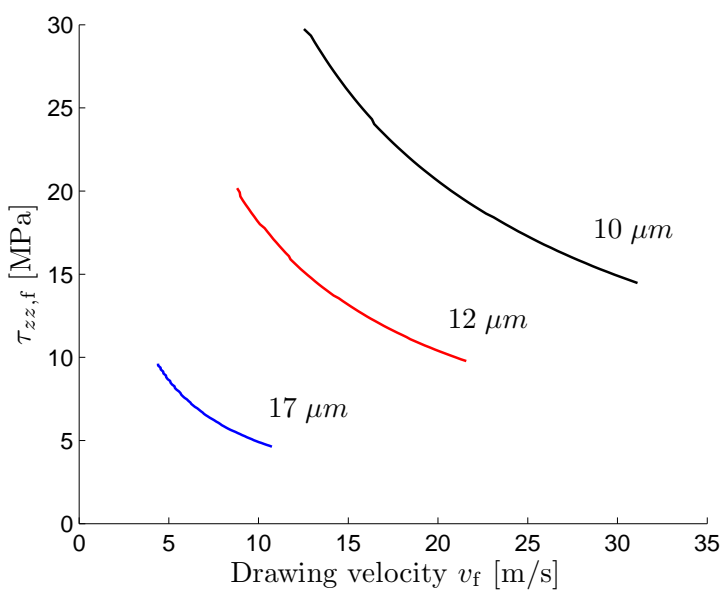

Fig. 8 Final axial stress $\tau_{z z, \mathrm{f}}$ as a function of the winder velocity $v_{\mathrm{f}}$ while keeping the final diameter constant. For each curve, the range of $T_{0}$ is $[1250-1310]^{\circ} \mathrm{C}$ (the highest temperature corresponds to the lower right end of each curve). 


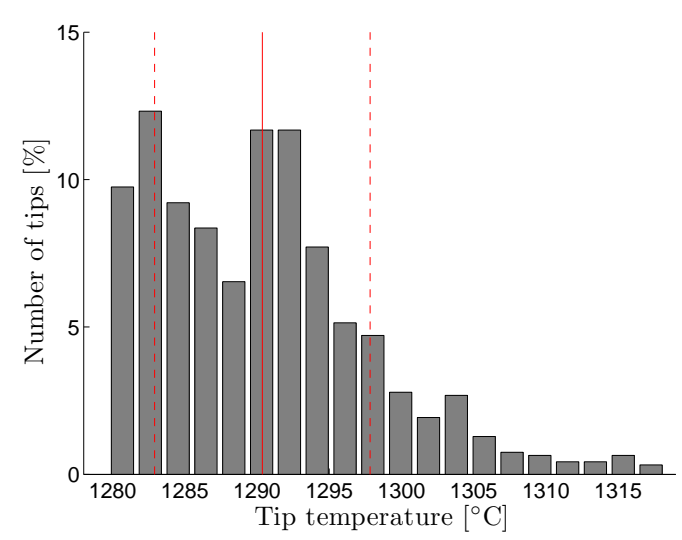

(a)

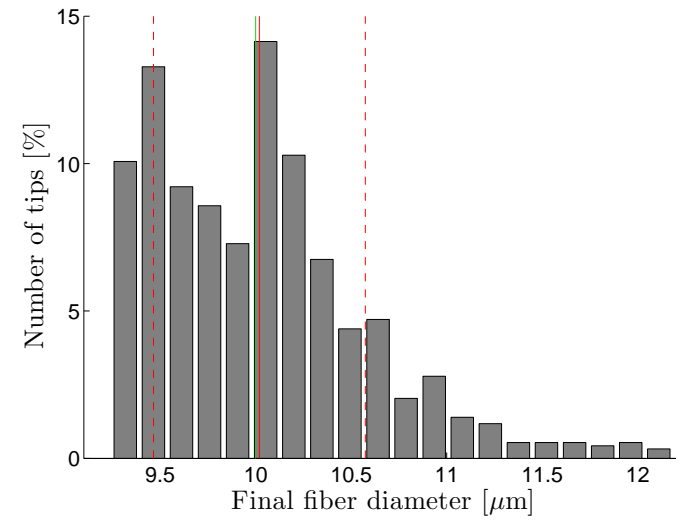

(b)

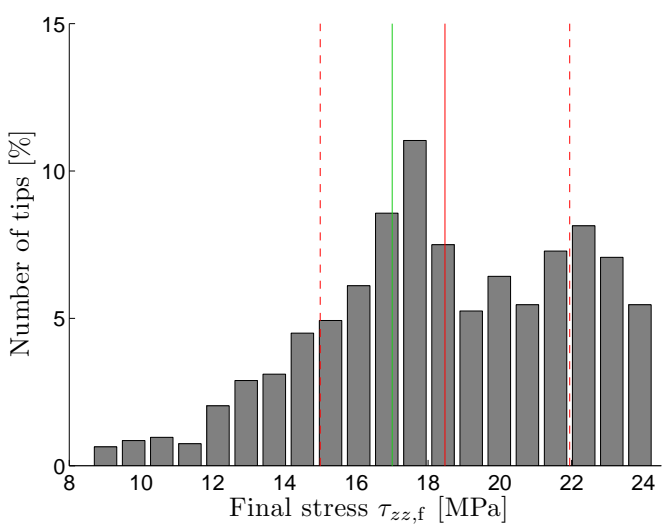

(c)

Fig. 9 (a) Distribution of the tip temperature $T_{0}$ for a bushing plate with 6000 fibers (from [12]). (b) Final fiber diameter resulting from the temperature distribution shown in (a). (c) Axial stress $\tau_{z z, \mathrm{f}}$ resulting from the temperature distribution shown in (a). The mean and the standard deviation are indicated by the continuous and dashed red lines, respectively. The green line indicates the value corresponding to the mean temperature.

a large drawing velocity whereas equation (22) and previous analysis seems to predict the opposite. This can be explained by the largest impact of a high temperature $T_{0}$ that reduces the stress. As a result, increasing the bushing temperature and the drawing velocity accordingly (i.e., increasing the flow rate) allows to reduce the stress levels in the fiber. Unfortunately, this optimization strategy is limited because a too high tip temperature leads to hydrodynamic instabilities preventing the drawing of the fiber [5].

\subsection{Temperature inhomogeneity of the bushing plate}

Previous sections considered a single fiber. However, in the industrial process the bushing plate is typically composed of thousands of fibers. Although each of these fibers is drawn at the same velocity $v_{\mathrm{f}}$, their corresponding tip temperature $T_{0}$ can strongly vary due to spatial temperature gradients of the bushing plate. These variations of $T_{0}$ across fibers lead to variations in the flow rates and, consequently, to a broad distribution of final fiber diameters around the target value.

This effect is quantified here for a bushing plate with 6000 fibers. The spatial temperature inhomogeneities of the bushing plate are obtained from numerical simulations [12], as illustrated in Figure 9a. In this case, the temperature spread is about $40^{\circ} \mathrm{C}$, with most fibers in the lower temperature range. This temperature distribution leads to the distribution of final diameter and stress shown in Figures $9 \mathrm{~b}-\mathrm{c}$. The final diameter ranges 
from $9.2 \mu \mathrm{m}$ to $12.1 \mu \mathrm{m}$, and the stress from $9 \mathrm{MPa}$ to $24 \mathrm{MPa}$. The mean of the distribution and one standard deviation from the mean are indicated by the continuous and dashed red lines, respectively. In Figures $9 \mathrm{~b}-\mathrm{c}$, the green line corresponds to the final diameter and axial stress for the mean temperature. While the mean radius is almost identical to the radius of the mean temperature, this is not the case for the stress, highlighting the very nonlinear dependence of the stress on the temperature. This distribution illustrates the large range of stress for a same bushing position. Note that these variations are larger than those observed in section 4.2.

During production, the break of a single fiber causes the entire bushing position to shut down. Therefore, in order to reduce the breaking rate, the tails of the distribution should be reduced: in the lower $T_{0}$ range to minimize the stress, and in the higher range to avoid hydrodynamic instabilities. This analysis demonstrates the importance of reducing as much as possible any temperature inhomogeneity at the bushing plate.

\section{CONCLUSIONS}

The process of continuous glass fiber drawing involves many physical phenomena such as fluid dynamics and heat transfers. In particular, the glass flow is highly dependent on the temperature field through the nonconstant viscosity. The process is investigated here through numerical simulations from the perspective of industrial production. In particular, it is attempted to identify strategies that minimize the fiber stress so as to reduce the fiber breaking rate.

Both a two-dimensional axisymmetric model and a semi-analytical one-dimensional model are considered here. In the latter case, the forming fiber can be modeled as a pure extensional flow where all variables are solely a function of the axial component. It is shown that the one-dimensional model is computationally much more efficient and leads to results accurate enough for the optimization of the process operating window. It is therefore used for different sensitivity analyses. Nevertheless, radial effects can be important near the tip exit and the one-dimensional approximation should be used with care in this region.

The effect of physical parameters controlling heat transfers, such as the radiative emissivity, the convective heat transfer or the environment air temperature, have been investigated. Overall, it is found that larger heat transfers lead to a higher axial stress. The semi-analytical model shows the dependance of the stress on the cooling through the viscosity variation over the whole domain. In particular, the viscosity at the tip exit has a strong effect on the stress.

The subsequent sensitivity analysis on the process parameters also demonstrates that the key parameter is the glass temperature at the tip. Additionally, it is shown that the stress for a given final diameter can be minimized by increasing the tip temperature and drawing speed. However, this strategy is limited by hydrodynamic instabilities that start to form when the temperature is too high. As a consequence, the spatial variations of temperature over the bushing plate are very critical as they lead to a broad distribution of stress. This illustrates the importance of considering the entire bushing plate in the study of the process, and not simply a single fiber.

Future work will focus on improving the physical modeling by considering internal radiation, and on characterizing the stress leading to the failure of the fiber. A special attention will be paid on the convective coefficient in case of non constant ambient temperature. In addition, unsteady effects will be investigated to identify the limits of instabilities.

\section{ACKNOWLEDGMENTS}

The authors would like to acknowledge the financial and technical support of 3B-the Fibreglass company, Binani Group, and the financial support of the Walloon Region. 


\section{REFERENCES}

[1] Glicksman, L.R. An investigation of the shape, temperature distribution and tension of a heated free jet flowing at ultra low reynolds number. PhD Thesis, Massachusetts Institute of Technology (1964).

[2] Glicksman, L.R., The dynamics of a heated free jet of variable viscosity liquid at low reynolds numbers, Journal of Basic Engineering 90, 3:343-354 (1968).

[3] Huynh, B.P. and Tanner, R.I., Study of the non-isothermal glass fibre drawing process, Rheologica Acta 22, 5:482499 (1983).

[4] Purnode, B.A. and Rubin, Y., Two-dimensional Finite Element Analysis of Glass Fiber Forming, 8th International Congress on Glass, San Fransisco, USA (1998).

[5] Purnode, B.A., Transient Axisymmetric Study of Glass Fiber Forming, Proceedings of ASME 2000 Fluids Engineering Division Summer Meeting, Boston, USA (2000).

[6] Von Der Ohe, R., Simulation of Glass Fiber Forming Processes, Phd Thesis, Aalborg University, Denmark (2003).

[7] Rekhson, S., Leonard, J. and Sanger, P., Attenuation and Breakage in the Continuous Glass Fiber Drawing Process, Ceram. Eng. Sci. Proc. 25, 1:179-190 (2004).

[8] Rekhson, S., Improvement of performance and yield of continuous glass fiber drawing technology, Proceedings of the FY 2005 Glass, Chicago, USA (2005).

[9] Liu, S. and Banta, L.E., Parametric Study of Glass Fiber Drawing Process, International Journal of Applied Glass Science 1, 2:180-187 (2010).

[10] Loewenstein, K.L., The Manufacturing Technology of Continuous Glass Fibers, Elsevier Scientific Publishing Company, New York (1973).

[11] Kase, S. and Matsuo, T.J., Studies of melt spinning I, fundamental equations on the dynamics of melt spinning, Journal of Polymer science, Part A: General papers 3, 7:2541-2554 (1965).

[12] Ozden, U.A., Numerical simulation of the tip plate temperature gradient in a glassfiber bushing during production, 1st International Glass Fiber Symposia, Aachen, Germany (2012)

[13] Chouffart Q., Simon P. and Terrapon V.E., Numerical investigation of continuous fiber glass drawing, Proceedings of the V International Conference on Computational Methods for Coupled Problems in Science and Engineering, Santa Eulalia, Ibiza (2013)

[14] Crochet, M.J., Keunings R., Debbaut B., and Marchal J.M., Polyflow: a multi-purpose finite element program for continuous polymer flows, Computer Modeling for Extrusion and Other Continuous Polymer Processes, 25-50 (1992).

[15] Sweetland, M., Nozzle Analysis and Heat Transfer Model for Spray Cooling of Glass Fibers, Master Thesis, Massachusetts Institute of Technology, USA (1998). 